\title{
Comparing laser and polychromatic confocal optical displacement sensors for the 3-D measurement of cylindrical artefacts containing microscopic grooved structures
}

\author{
P.J. Boltryk ${ }^{*}$, M. Hill and J.W. McBride \\ Electro-Mechanical Research Group, School of Engineering Sciences, University of Southampton, Southampton, SO17 1BJ, UK
}

Received Date Line (to be inserted by Production) ( $8 \mathrm{pt}$ )

\begin{abstract}
A scanning system has been developed for measuring the surface of early cylinder mechanical sound recordings to high precision, both for surface preservation and for post-processing of the data to recover the sound encoded in the grooves. Research has identified that high sensor axial resolution is required to resolve the smallest amplitude groove modulations contained on typical cylinder artefacts resulting in the selection of confocal laser and white light (polychromatic) confocal sensors as suitable sensing technologies. This paper presents data acquired by the two confocal sensor technologies, comparing the measured surface profiles with data obtained using a stylus profilometer. We investigate limitations of the sensors, relating their characteristics to the ongoing development of the scanning system.
\end{abstract}

Keywords: non-contact metrology, optical displacement sensor, profilometer, sound recovery

\section{Introduction}

Single-point optical displacement sensors coupled with a scanning system provide a common means for noncontact measurement of complex surface profiles. The research presented in this paper falls within a number of research programmes that have applied precision metrology to the measurement of early sound recording artefacts (see, for example, [1]-[4]). Worldwide, sound archives maintain collections of cylinder and flat disc media, which due to their material properties are fragile, and structurally degrading due to aging effects and biological problems such as mould growth. Non-contact surface mapping provides opportunities for preservation through providing a permanent digital record of the surface for posterity, and also for sound recovery using post-measurement processing of the surface data. This process also allows badly deformed recordings to be played, where stylus playback would not be possible. Extremely high sensor axial resolution is required to resolve the smallest amplitude groove modulations contained on typical cylinder artefacts [5]. For this reason, the project has selected confocal laser and white light (polychromatic) confocal sensors as suitable sensing technologies, and rejected the popular laser triangulation sensor for this application based on its inability to resolve typical groove cross-sections and its more limited axial resolution. Since mechanical sound carriers encode the sound in sound-carrying grooves, which, by definition are composed of angled features, optimising the scanning system for performance

*Corresponding author. Tel.: +44 (0)23 8059 2339; fax: +44 (0)23 80593053.

E-mail address: boltryk@soton.ac.uk (P.J. Boltryk) 
on inclined surfaces is of paramount importance. Therefore, despite the high z-axis resolution capability of white light interferometers, we discount this technique as tests on a sample cylinder have demonstrated unacceptably high levels of data-loss on the inclined surfaces.

This paper presents data acquired by the two confocal sensor technologies, comparing the measured surface profiles with data obtained using a stylus profilometer. We investigate differences in surface profiles obtained by the two sensing methods, making reference to factors such as the system's scanning speed, the sensor's spot diameter, and the elongated spot diameter caused by the raster movement of the scanning system.

\section{System details}

The cylindrical artefact is mounted on a mandrel between conical centres. The mandrel itself is rotated to high resolution through full $360^{\circ}$ using a rotational stage. An optical displacement sensor is traversed along the axis of the cylinder, which measures the radial height of the surface of the cylinder along the linescan. The distance from the surface to the sensor, together with the sensor's standoff distance is used to characterise the height of the surface. The artefact is incremented through a specified angular rotation between consecutive linescans, such that the whole surface is measured. The resultant dataset of $(x, y, z)$ measurements represents the unwrapped cylinder's surface topology. A more detailed description of the system is presented in [6]. Image and signal processing techniques may be applied post-measurement to recover the audio content of the cylinder [6], [7], allowing access to the cultural content of the recordings.

\section{Specific system metrology demands}

Sound encoded in the grooves as displacement modulations have been observed to contain spectra with sub$50 \mathrm{~nm}$ amplitudes [5]. To resolve displacement modulations to this scale requires a high resolution displacement sensor, with a recommended axial resolution of at least $10-20 \mathrm{~nm}$. In common with other metrology applications, the compromise between depth of field of the optical sensor (expressed as the sensor's gauge range) versus axial resolution presents a particular problem for this scanning process, because the cylinder artefacts tend to exhibit non-concentricity caused by creep and long-term storage.

A second major challenge for the metrology system is the large surface area to be scanned. The recorded tracks on both 2 and 4 minute long cylinders are typically about $95 \mathrm{~mm}$ long with diameter $55 \mathrm{~mm}$, resulting in an unwrapped surface area of $16415 \mathrm{~mm}^{2}$. The grooves on typical Blue Amberol cylinder recordings are about $15 \mu \mathrm{m}$ deep and have a pitch of $130 \mu \mathrm{m}$ and $260 \mu \mathrm{m}$ for 4 minute and 2 minute recordings, respectively. To resolve the groove structure requires a linescan resolution of at least $10 \mu \mathrm{m}$ spatial sampling across the grooves for the $130 \mu \mathrm{m}$ pitch cylinders. Each linescan, therefore, requires at least 9500 datapoints. Since the reconstruction of the playback stylus trajectory for audio recovery requires $96 \mathrm{kHz}$ sampling of the grooves for data redundancy, equating to an angular increment of $0.01^{\circ}$ between consecutive linescans, the scanning of the cylinder media therefore requires approximately $0.34 \times 10^{9}$ datapoints. Researching methods for minimising the scan time (increasing sensor sampling rates, for example) is therefore of paramount importance for system viability.

\section{Sensor developments}

In [5], the relative performances of commercial WL and CL sensors were compared on a reference cylinder machined with grooves, and on a 4 minute Blue Amberol cylinder recording (cylinder reference \#1). A common observation when comparing groove cross-sections as measured by the two competing sensors was the slight discrepancy in implied shape for nominally identical grooves. Fig. 1(a) plots the profiles as measured by the two sensors, for a short $1 \mathrm{~mm}$ length at the end of the track, with the uncut end of the cylinder evident towards the right hand side of the profiles. The profiles illustrate that the polychromatic sensor tended to suggest more rounded 
groove tops, whilst the peaks of the grooves measured by the confocal laser sensor were sharper. The WL sensor had a $7 \mu \mathrm{m}$ spot diameter, axial resolution of $10 \mathrm{~nm}$, and acquired data at a rate of $1000 \mathrm{~Hz}$, whilst the CL sensor had a spot size of $2 \mu \mathrm{m}$, system data acquisition rate of $82 \mathrm{~Hz}$ (16 averaged measurements for each measurement data point, with CL sensor obtaining each single measurement at $1.4 \mathrm{kHz}$ ) and an axial resolution of $100 \mathrm{~nm}$. An advantage of the CL sensor is the gauge range of $600 \mu \mathrm{m}$, compared with $350 \mu \mathrm{m}$ for the WL sensor, making it easier to implement on the non-concentric cylinders. The two sensors are compared with a reference stylus profilometer, using $0.25 \mu \mathrm{m}$ lateral sampling resolution and a $2 \mu \mathrm{m}$ sapphire stylus.
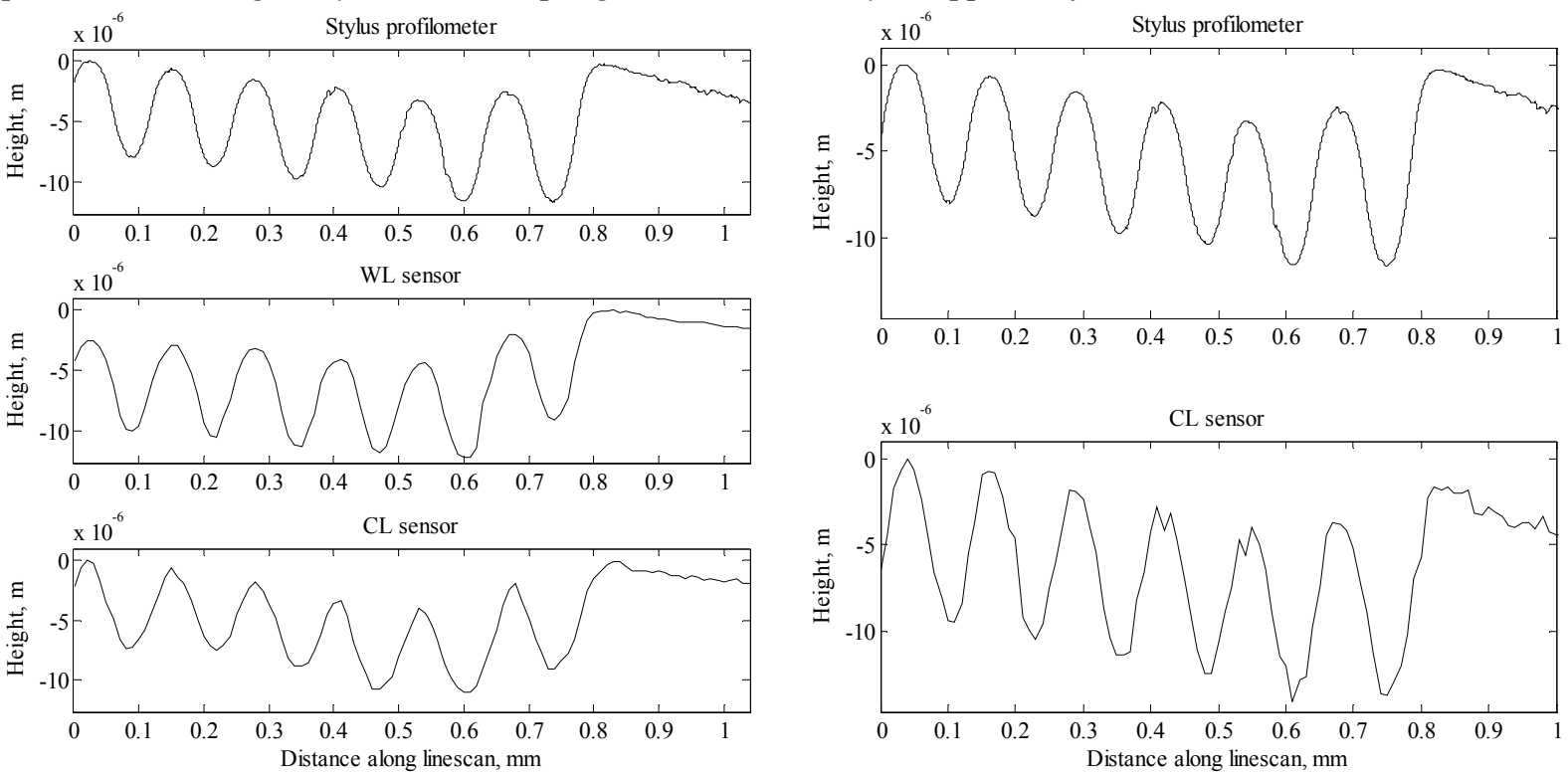

Fig. 1 (a) Groove cross-sections (cylinder reference \#1) as measured by stylus profilometer (top axis), WL sensor (middle axis) and CL sensor (bottom axis). (b) Groove cross-sections as measured by stylus profilometer (top axis) and higher-resolution CL sensor (bottom axis). Scanning system samples at $10 \mu \mathrm{m}$ resolution along the linescan axis using both optical sensors, and at $0.25 \mu \mathrm{m}$ for the stylus profilometer.

This paper addresses the use of a replacement commercial CL sensor, with a higher axial resolution of 10nm that matches the specified WL sensor's axial resolution, and also the use of a WL sensor with smaller 3.5 $\mu \mathrm{m}$ spot size capable of operating up to $4 \mathrm{kHz}$.

\section{Profiles obtained using replacement commercial CL sensor with 10nm axial resolution}

Fig. 1(b) demonstrates the agreement between the measured profiles obtained using the replacement CL sensor and the reference stylus profilometer, when compared with the older, lower resolution CL results presented in fig. 1(a). This can be observed in the tops of the groove structures which are smoother in fig. 1(b). A 2 minute Blue Amberol cylinder, reference \#2, has also been investigated, the results for which are plotted in fig.2. This cylinder has a more irregular surface than the smooth grooves exhibited by cylinder reference \#1, which might be attributed to biological growth. In the presence of this higher apparent surface roughness, the surfaces as measured by the two optical sensors are reasonably consistent in shape.

Referring to fig. 2, the CL sensor suffers from more data loss on inclined surfaces than the WL sensor (observed as gaps in the profiles for all plots), but this is generally improved when a 16-point average is used. However, using a 16-point average drastically reduces the possible system data acquisition rates from $454 \mathrm{~Hz}(2-$ point average) to $82 \mathrm{~Hz}$, negating efforts to reduce the scan time for cylinders. In addition, the averaging process 
effectively elongates the light spot area addressed by the sensor as it takes a given measurement which tends to smooth out smaller features. For example, using 16-point average, the system traverses the sensor at a speed of $0.56 \mathrm{~mm} / \mathrm{s}$, resulting in a length addressed by the spot of $6.8 \mu \mathrm{m}$ during the $82 \mathrm{~Hz}$ sampling period. Fig. 2 also demonstrates the occurrence of spurious outliers for the CL sensor, an important characteristic of CL data, which are more prevalent when operating with 2-point average. Such data artefacts affect the sound recovery procedure because, uncorrected, can introduce impulsive noise to the audio signal.
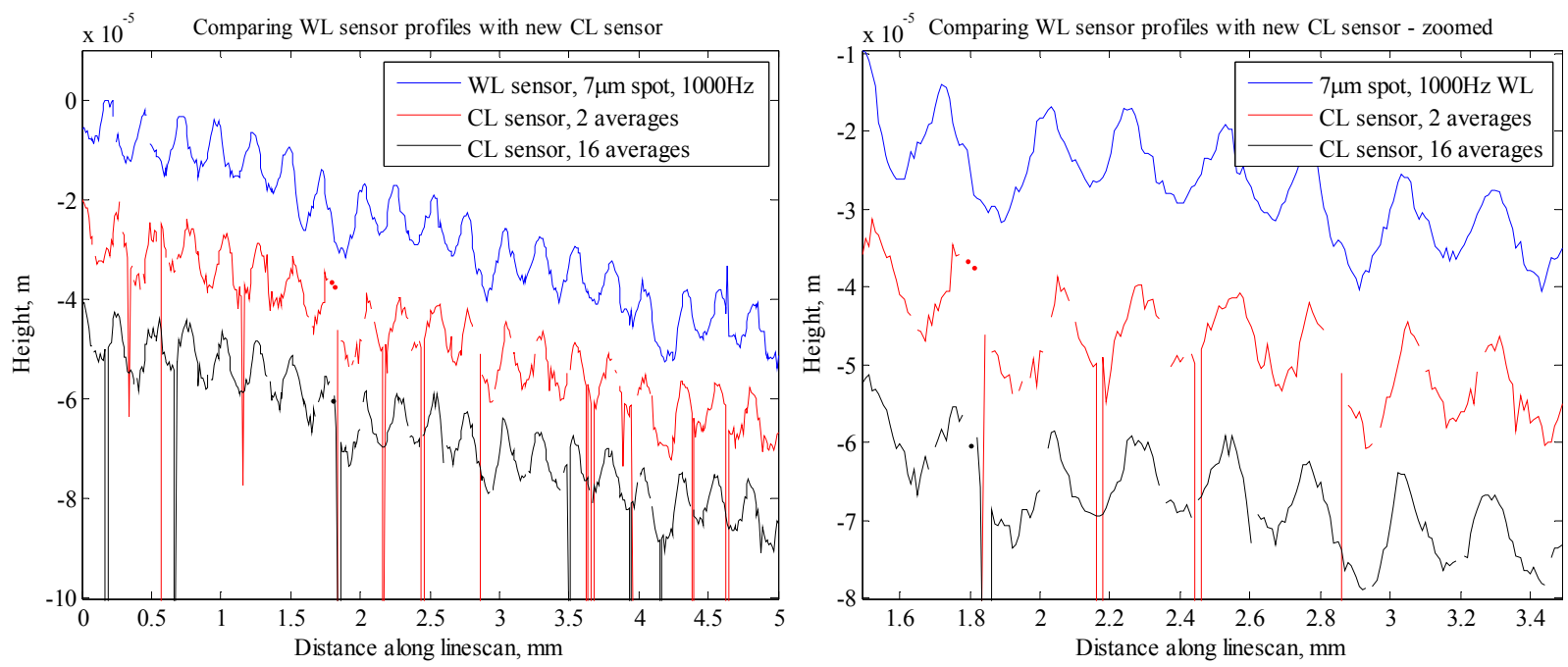

Fig. 2. (a) Profile obtained by WL sensor operating at 1000 with a $7 \mu \mathrm{m}$ spot size, compared with replacement CL sensor profiles obtained using 2-point average and 16-point average. (b) Zoomed view for same profiles. Scanning system samples at $10 \mu \mathrm{m}$ resolution along the linescan axis using both sensors. Scanned cylinder reference \#2. For clarity in both plots, ordinate axis offsets have been adjusted such that the top profile is that measured by the WL sensor, the middle profile results from the

replacement CL sensor operating with 2 averages and the lower profile results from the CL sensor, but using a 16-point average

\section{Limitations of WL sensor spot size and sample frequency}

The benefits offered by using an upgraded WL sensor with $3.5 \mu \mathrm{m}$ spot size and a higher potential operating frequency include the ability to resolve smaller features (in the lateral sense), and reduced scan times for the cylinders due to higher data acquisition rates. However, fig. 3(a) illustrates that using a 3.5 $\mu \mathrm{m}$ spot size makes the sensor more susceptible to inclined surfaces (higher levels of data loss), due to a reduced quantity of backreflected light. It is usual to reduce the sampling frequency to improve performance on inclined surfaces, which has the effect of increasing the net light quantity received by the sensor's spectrometer during a given measurement cycle. However, these results suggest that despite reducing the sampling frequency to $200 \mathrm{~Hz}$, the data loss for the $3.5 \mu \mathrm{m}$ spot size exceeds that obtained by a WL sensor using a $7 \mu \mathrm{m}$ spot, operating at $1000 \mathrm{~Hz}$.

Reverting to a $7 \mu \mathrm{m}$ spot, the effect of sample frequency on data loss is investigated. A small $1 \mathrm{~mm} \mathrm{x}{ }^{1 / 4}{ }^{\circ}$ area of the surface was scanned at a series of sensor operating frequencies, between 400 and $4 \mathrm{kHz}$. At each frequency, 10 repeat areal measurements were made and the percentage of bad data calculated. The results presented in fig. 3(b) highlight that even with a $7 \mu \mathrm{m}$ spot, for a preservation record of the surface where minimal data is acceptable, the system is limited to the sensor operating at $1200 \mathrm{~Hz}$. 

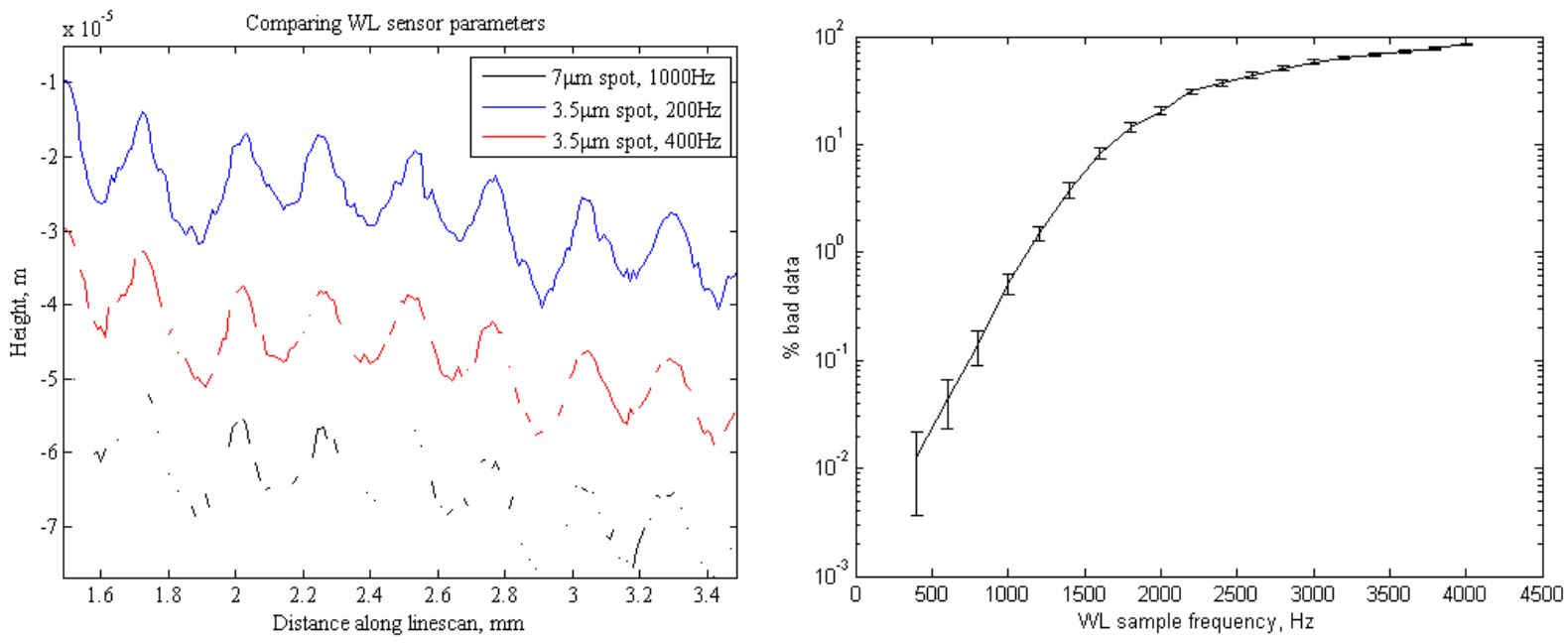

Fig. 3. (a) Profile obtained by WL sensor operating at 1000 with a $7 \mu \mathrm{m}$ spot size, compared with replacement WL sensor using a $3.5 \mu \mathrm{m}$ spot, operating at $200 \mathrm{~Hz}$ and $400 \mathrm{~Hz}$. Scanning system samples at $10 \mu \mathrm{m}$ resolution along the linescan axis using both sensors. Scanned cylinder reference \#2. For clarity, ordinate axis offsets have been adjusted to separate profiles. (b) Percentage of bad data recorded as a function of sensor sampling frequency. The data was averaged across 10 repeat measurements at each frequency, with the error bars representing the range.

\section{Conclusions}

Previous observations concerning inconsistencies between profiles measured by WL and CL sensors have been overcome through use of an updated CL sensor. However, with scan time being a crucial factor for making the system feasible, the WL sensor remains the sensor of choice because the system can achieve higher data acquisition rates. Attempts to improve upon the WL sensor, through use of smaller spot size and higher potential sample frequencies have highlighted potential physical limitations of this sensing technology on the surfaces encountered in early mechanical recording media.

\section{Acknowledgements}

The authors gratefully acknowledge support from the UK's Engineering and Physical Sciences Research Council, the British Library Sound Archive and TaiCaan Technologies Ltd.

\section{References}

[1] Petrov, Kryuchin, et al., Optomechanical Method of Sound from Edison Cylinders. International Conference on Optical Storage, Imaging and Transmission of Information (1997).

[2] Shanoylo, Kosyak, et al., Reading and processing of audio information reproduced from Edison phonograph cylinders by method of laser interferometry. Laser Techniques and Systems in Art Conservation (2001).

[3] Penn, W. A. and M. J. Hanson, The Syracuse University Library Radius Project: Development of a non-destructive playback system for cylinder recordings." First Monday 8(5) (2003).

[4] Asakura, T. and J. Uozumi, Optical methods for Reproducing Sounds from Old Phonograph Records. International Trends in Optics and Photonics ICO IV: 65-81 (1999).

[5] P.J. Boltryk , M. Hill, J.W. McBride and A. Nasce, A comparison of precision optical displacement sensors for the 3D measurement of complex surface profiles', Sens. Actuators A: Phys. (2007), doi:10.1016/j.sna.2007.03.006

[6] V. Fadeyev, C. Haber, C. Maul, J.W. McBride, M. Golden; Journal of the Audio Engineering Society, 53, (6), (2005), 485-508.

[7] A. Nasce, J.W. McBride, M. Hill and P.J. Boltryk, 'Signal processing methods for the recovery of audio from early acoustic cylinder recordings, measured via non-contact optical sensor', accepted for presentation at AES 31st International Conference, London, UK, June 25-27, 2007. 